\title{
Stable Signal Recovery from Incomplete and Inaccurate Measurements
}

\author{
Emmanuel Candes ${ }^{\dagger}$, Justin Romberg ${ }^{\dagger}$, and Terence Tao ${ }^{\sharp}$ \\ $\dagger$ Applied and Computational Mathematics, Caltech, Pasadena, CA 91125 \\ $\sharp$ Department of Mathematics, University of California, Los Angeles, CA 90095
}

February, 2005; Revised June 2005

\begin{abstract}
Suppose we wish to recover a vector $x_{0} \in \mathbb{R}^{m}$ (e.g. a digital signal or image) from incomplete and contaminated observations $y=A x_{0}+e ; A$ is a $n$ by $m$ matrix with far fewer rows than columns $(n \ll m)$ and $e$ is an error term. Is it possible to recover $x_{0}$ accurately based on the data $y$ ?

To recover $x_{0}$, we consider the solution $x^{\sharp}$ to the $\ell_{1}$-regularization problem

$$
\min \|x\|_{\ell_{1}} \text { subject to }\|A x-y\|_{\ell_{2}} \leq \epsilon,
$$

where $\epsilon$ is the size of the error term $e$. We show that if $A$ obeys a uniform uncertainty principle (with unit-normed columns) and if the vector $x_{0}$ is sufficiently sparse, then the solution is within the noise level

$$
\left\|x^{\sharp}-x_{0}\right\|_{\ell_{2}} \leq C \cdot \epsilon .
$$

As a first example, suppose that $A$ is a Gaussian random matrix, then stable recovery occurs for almost all such $A$ 's provided that the number of nonzeros of $x_{0}$ is of about the same order as the number of observations. As a second instance, suppose one observes few Fourier samples of $x_{0}$, then stable recovery occurs for almost any set of $n$ coefficients provided that the number of nonzeros is of the order of $n /[\log m]^{6}$.

In the case where the error term vanishes, the recovery is of course exact, and this work actually provides novel insights on the exact recovery phenomenon discussed in earlier papers. The methodology also explains why one can also very nearly recover approximately sparse signals.
\end{abstract}

Keywords. $\ell_{1}$-minimization, basis pursuit, restricted orthonormality, sparsity, singular values of random matrices.

Acknowledgments. E. C. is partially supported by a National Science Foundation grant DMS 01-40698 (FRG) and by an Alfred P. Sloan Fellowship. J. R. is supported by National Science Foundation grants DMS 01-40698 and ITR ACI-0204932. T. T. is supported in part by grants from the Packard Foundation. 


\section{Introduction}

\subsection{Exact recovery of sparse signals}

Recent papers $[2-5,10]$ have developed a series of powerful results about the exact recovery of a finite signal $x_{0} \in \mathbb{R}^{m}$ from a very limited number of observations. As a representative result from this literature, consider the problem of recovering an unknown sparse signal $x_{0}(t) \in \mathbb{R}^{m}$; that is, a signal $x_{0}$ whose support $T_{0}=\left\{t: x_{0}(t) \neq 0\right\}$ is assumed to have small cardinality. All we know about $x_{0}$ are $n$ linear measurements of the form

$$
y_{k}=\left\langle x_{0}, a_{k}\right\rangle \quad k=1, \ldots, n \quad \text { or } \quad y=A x_{0},
$$

where the $a_{k} \in \mathbb{R}^{m}$ are known test signals. Of special interest is the vastly underdetermined case, $n \ll m$, where there are many more unknowns than observations. At first glance, this may seem impossible. However, it turns out that one can actually recover $x_{0}$ exactly by solving the convex program ${ }^{1}$

$$
\left(P_{1}\right) \quad \min \|x\|_{\ell_{1}} \quad \text { subject to } A x=y,
$$

provided that the matrix $A \in \mathbb{R}^{n \times m}$ obeys a uniform uncertainty principle.

The uniform uncertainty principle, introduced in [5] and refined in [4], essentially states that the $n \times m$ measurement matrix $A$ obeys a "restricted isometry hypothesis." To introduce this notion, let $A_{T}, T \subset\{1, \ldots, m\}$ be the $n \times|T|$ submatrix obtained by extracting the columns of $A$ corresponding to the indices in $T$. Then [4] defines the $S$-restricted isometry constant $\delta_{S}$ of $A$ which is the smallest quantity such that

$$
\left(1-\delta_{S}\right)\|c\|_{\ell_{2}}^{2} \leq\left\|A_{T} c\right\|_{\ell_{2}}^{2} \leq\left(1+\delta_{S}\right)\|c\|_{\ell_{2}}^{2}
$$

for all subsets $T$ with $|T| \leq S$ and coefficient sequences $\left(c_{j}\right)_{j \in T}$. This property essentially requires that every set of columns with cardinality less than $S$ approximately behaves like an orthonormal system. It was shown (also in [4]) that if $S$ verifies

$$
\delta_{S}+\delta_{2 S}+\delta_{3 S}<1
$$

then solving $\left(P_{1}\right)$ recovers any sparse signal $x_{0}$ with support size obeying $\left|T_{0}\right| \leq S$.

\subsection{Stable recovery from imperfect measurements}

This paper develops results for the "imperfect" (and far more realistic) scenarios where the measurements are noisy and the signal is not exactly sparse. Everyone would agree that in most practical situations, we cannot assume that $A x_{0}$ is known with arbitrary precision. More appropriately, we will assume instead that one is given "noisy" data $y=A x_{0}+e$, where $e$ is some unknown perturbation bounded by a known amount $\|e\|_{\ell_{2}} \leq \epsilon$. To be broadly applicable, our recovery procedure must be stable: small changes in the observations should result in small changes in the recovery. This wish, however, may be quite hopeless. How can we possibly hope to recover our signal when not only the available information is severely incomplete but in addition, the few available observations are also inaccurate?

\footnotetext{
${ }^{1}\left(P_{1}\right)$ can even be recast as a linear program [6].
} 
Consider nevertheless (as in [12] for example) the convex program searching, among all signals consistent with the data $y$, for that with minimum $\ell_{1}$-norm

$$
\left(P_{2}\right) \quad \min \|x\|_{\ell_{1}} \quad \text { subject to } \quad\|A x-y\|_{\ell_{2}} \leq \epsilon .
$$

The first result of this paper shows that contrary to the belief expressed above, the solution to $\left(P_{2}\right)$ recovers an unknown sparse object with an error at most proportional to the noise level. Our condition for stable recovery again involves the restricted isometry constants.

Theorem 1 Let $S$ be such that $\delta_{3 S}+3 \delta_{4 S}<2$. Then for any signal $x_{0}$ supported on $T_{0}$ with $\left|T_{0}\right| \leq S$ and any perturbation e with $\|e\|_{\ell_{2}} \leq \epsilon$, the solution $x^{\sharp}$ to $\left(P_{2}\right)$ obeys

$$
\left\|x^{\sharp}-x_{0}\right\|_{\ell_{2}} \leq C_{S} \cdot \epsilon,
$$

where the constant $C_{S}$ may only depend on $\delta_{4 S}$. For reasonable values of $\delta_{4 S}, C_{S}$ is well behaved; e.g. $C_{S} \approx 8.82$ for $\delta_{4 S}=1 / 5$ and $C_{S} \approx 10.47$ for $\delta_{4 S}=1 / 4$.

It is interesting to note that for $S$ obeying the condition of the theorem, the reconstruction from noiseless data is exact. It is quite possible that for some matrices $A$, this condition tolerates larger values of $S$ than (31).

We would like to offer two comments. First, the matrix $A$ is rectangular with many more columns than rows. As such, most of its singular values are zero. As emphasized earlier, the fact that the severely ill-posed matrix inversion keeps the perturbation from "blowing up" is rather remarkable and perhaps unexpected.

Second, no recovery method can perform fundamentally better for arbitrary perturbations of size $\epsilon$. To see why this is true, suppose one had available an oracle letting us know, in advance, the support $T_{0}$ of $x_{0}$. With this additional information, the problem is well-posed and one could reconstruct $x_{0}$ by the method of Least-Squares for example,

$$
\hat{x}= \begin{cases}\left(A_{T_{0}}^{*} A_{T_{0}}\right)^{-1} A_{T_{0}}^{*} y & \text { on } T_{0} \\ 0 & \text { elsewhere. }\end{cases}
$$

In the absence of any other information, one could easily argue that no method would exhibit a fundamentally better performance. Now of course, $\hat{x}-x_{0}=0$ on the complement of $T_{0}$ while on $T_{0}$

$$
\hat{x}-x_{0}=\left(A_{T_{0}}^{*} A_{T_{0}}\right)^{-1} A_{T_{0}}^{*} e,
$$

and since by hypothesis, the eigenvalues of $A_{T_{0}}^{*} A_{T_{0}}$ are well-behaved ${ }^{2}$

$$
\left\|\hat{x}-x_{0}\right\|_{\ell_{2}} \approx\left\|A_{T_{0}}^{*} e\right\|_{\ell_{2}} \approx \epsilon,
$$

at least for perturbations concentrated in the row space of $A_{T_{0}}$. In short, obtaining a reconstruction with an error term whose size is guaranteed to be proportional to the noise level is the best one can hope for.

Remarkably, not only can we recover sparse input vectors but one can also stably recover approximately sparse vectors, as we have the following companion theorem.

\footnotetext{
${ }^{2}$ Observe the role played by the singular values of $A_{T_{0}}$ in the analysis of the oracle error.
} 
Theorem 2 Suppose that $x_{0}$ is an arbitrary vector in $\mathbb{R}^{m}$ and let $x_{0, S}$ be the truncated vector corresponding to the $S$ largest values of $x_{0}$ (in absolute value). Under the hypothesis of Theorem [1, the solution $x^{\sharp}$ to $\left(P_{2}\right)$ obeys

$$
\left\|x^{\sharp}-x_{0}\right\|_{\ell_{2}} \leq C_{1, S} \cdot \epsilon+C_{2, S} \cdot \frac{\left\|x_{0}-x_{0, S}\right\|_{\ell_{1}}}{\sqrt{S}} .
$$

For reasonable values of $\delta_{4 S}$ the constants in (5D) are well behaved; e.g. $C_{1, S} \approx 12.04$ and $C_{1, S} \approx 8.77$ for $\delta_{4 S}=1 / 5$.

Roughly speaking, the theorem says that minimizing $\ell_{1}$ stably recovers the $S$-largest entries of an $m$-dimensional unknown vector $x$ from $n$ measurements only.

We now specialize this result to a commonly discussed model in mathematical signal processing, namely, the class of compressible signals. We say that $x_{0}$ is compressible if its entries obey a power law

$$
\left|x_{0}\right|_{(k)} \leq C_{r} \cdot k^{-r}
$$

where $\left|x_{0}\right|_{(k)}$ is the $k$ th largest value of $x_{0}\left(\left|x_{0}\right|_{(1)} \geq\left|x_{0}\right|_{(2)} \geq \ldots \geq\left|x_{0}\right|_{(m)}\right), r>1$, and $C_{r}$ is a constant which depends only on $r$. Such a model is appropriate for the wavelet coefficients of a piecewise smooth signal, for example. If $x_{0}$ obeys (7), then

$$
\frac{\left\|x_{0}-x_{0, S}\right\|_{\ell_{1}}}{\sqrt{S}} \leq C_{r}^{\prime} \cdot S^{-r+1 / 2} .
$$

Observe now that in this case

$$
\left\|x_{0}-x_{0, S}\right\|_{\ell_{2}} \leq C_{r}^{\prime \prime} \cdot S^{-r+1 / 2},
$$

and for generic elements obeying (7), there are no fundamentally better estimates available. Hence, we see that with $n$ measurements only, we achieve an approximation error which is almost as good as that one would obtain by knowing everything about the signal $x_{0}$ and selecting its $S$-largest entries.

As a last remark, we would like to point out that in the noiseless case, Theorem 2 improves upon an earlier result from Candès and Tao, see also [8]; it is sharper in the sense that 1) this is a deterministic statement and there is no probability of failure, 2) it is universal in that it holds for all signals, 3) it gives upper estimates with better bounds and constants, and 4) it holds for a wider range of values of $S$.

\subsection{Examples}

It is of course of interest to know which matrices obey the uniform uncertainty principle with good isometry constants. Using tools from random matrix theory, $[3,5,10]$ give several examples of matrices such that (3) holds for $S$ on the order of $n$ to within log factors. Examples include (proofs and additional discussion can be found in [5]):

- Random matrices with i.i.d. entries. Suppose the entries of $A$ are i.i.d. Gaussian with mean zero and variance $1 / n$, then $[5,10,17]$ show that the condition for Theorem 1 holds with overwhelming probability when

$$
S \leq C \cdot n / \log (m / n) .
$$


In fact, [4] gives numerical values for the constant $C$ as a function of the ratio $n / m$. The same conclusion applies to binary matrices with independent entries taking values $\pm 1 / \sqrt{n}$ with equal probability.

- Fourier ensemble. Suppose now that $A$ is obtained by selecting $n$ rows from the $m \times m$ discrete Fourier transform and renormalizing the columns so that they are unit-normed. If the rows are selected at random, the condition for Theorem 1 holds with overwhelming probability for $S \leq C \cdot n /(\log m)^{6}$ [5]. (For simplicity, we have assumed that $A$ takes on real-valued entries although our theory clearly accommodates complex-valued matrices so that our discussion holds for both complex and real-valued Fourier transforms.)

This case is of special interest as reconstructing a digital signal or image from incomplete Fourier data is an important inverse problem with applications in biomedical imaging (MRI and tomography), Astrophysics (interferometric imaging), and geophysical exploration.

- General orthogonal measurement ensembles. Suppose $A$ is obtained by selecting $n$ rows from an $m$ by $m$ orthonormal matrix $U$ and renormalizing the columns so that they are unit-normed. Then [5] shows that if the rows are selected at random, the condition for Theorem 1 holds with overwhelming probability provided

$$
S \leq C \cdot \frac{1}{\mu^{2}} \cdot \frac{n}{(\log m)^{6}}
$$

where $\mu:=\sqrt{m} \max _{i, j}\left|U_{i, j}\right|$. Observe that for the Fourier matrix, $\mu=1$, and thus (8) is an extension of the Fourier ensemble.

This fact is of significant practical relevance because in many situations, signals of interest may not be sparse in the time domain but rather may be (approximately) decomposed as a sparse superposition of waveforms in a fixed orthonormal basis $\Psi$; e.g. in a nice wavelet basis. Suppose that we use as test signals a set of $n$ vectors taken from a second orthonormal basis $\Phi$. We then solve $\left(P_{1}\right)$ in the coefficient domain

$$
\left(P_{1}^{\prime}\right) \quad \min \|\alpha\|_{\ell_{1}} \quad \text { subject to } \quad A \alpha=y,
$$

where $A$ is obtained by extracting $n$ rows from the orthonormal matrix $U=\Phi \Psi^{*}$. The recovery condition then depends on the mutual coherence $\mu$ between the measurement basis $\Phi$ and the sparsity basis $\Psi$ which measures the similarity between $\Phi$ and $\Psi$; $\mu(\Phi, \Psi)=\sqrt{m} \max \left|\left\langle\phi_{k}, \psi_{j}\right\rangle\right|, \phi_{k} \in \Phi, \psi_{j} \in \Psi$.

\subsection{Prior work and innovations}

The problem of recovering a sparse vector by minimizing $\ell_{1}$ under linear equality constraints has recently received much attention, mostly in the context of Basis Pursuit, where the goal is to uncover sparse signal decompositions in overcomplete dictionaries. We refer the reader to $[11,13]$ and the references therein for a full discussion.

We would especially like to note two works by Donoho, Elad, and Temlyakov [12], and Tropp [18] that also study the recovery of sparse signals from noisy observations by solving $\left(P_{2}\right)$ (and other closely related optimization programs), and give conditions for stable recovery. In [12], the sparsity constraint on the underlying signal $x_{0}$ depends on the magnitude of 
the maximum entry of the Gram matrix $M(A)=\max _{i, j: i \neq j}\left|\left(A^{*} A\right)\right|_{i, j}$. Stable recovery occurs when the number of nonzeros is at most $\left(M^{-1}+1\right) / 4$. For instance, when $A$ is a Fourier ensemble and $n$ is on the order of $m$, we will have $M$ at least of the order $1 / \sqrt{n}$ (with high probability), meaning that stable recovery is known to occur when the number of nonzeros is about at most $O(\sqrt{n})$. In contrast, the condition for Theorem 1 will hold when this number is about $n /(\log m)^{6}$, due to the range of support sizes for which the uniform uncertainty principle holds. In [18], a more general condition for stable recovery is derived. For the measurement ensembles listed in the previous section, however, the sparsity required is still on the order of $\sqrt{n}$ in the situation where $n$ is comparable to $m$. In other words, whereas these results require at least $O(\sqrt{m})$ observations per unknown, our results show that-ignoring log-like factors-only $O(1)$ are, in general, sufficient.

More closely related is the very recent work of Donoho [9] who shows a version of (5) in the case where $A \in \mathbb{R}^{n \times m}$ is a Gaussian matrix with $n$ proportional to $m$, with unspecified constants for both the support size and that appearing in (5). Our main claim is on a very different level since it is (1) deterministic (it can of course be specialized to random matrices), and (2) widely applicable since it extends to any matrix obeying the condition $\delta_{3 S}+3 \delta_{4 S}<2$. In addition, the argument underlying Theorem 1 is short and simple, giving precise and sharper numerical values. Finally, we would like to point out connections with fascinating ongoing work which develops fast randomized algorithms for sparse Fourier transforms $[14,19]$. Suppose $x_{0}$ is a fixed vector with $\left|T_{0}\right|$ nonzero terms, for example. Then [14] shows that it is possible to randomly sample the frequency domain $\left|T_{0}\right| \operatorname{poly}(\log m)$ times (poly $(\log m)$ denotes a polynomial term in $\log m)$, and reconstruct $x_{0}$ from these frequency data with positive probability. We do not know whether these algorithms are stable in the sense described in this paper, and whether they can be modified to be universal, i.e. reconstruct all signals of small support.

\section{Proofs}

\subsection{Proof of Theorem 1] sparse case}

The proof of the theorem makes use of two geometrical special facts about the solution $x^{\sharp}$ to $\left(P_{2}\right)$.

1. Tube constraint. First, $A x^{\sharp}$ is within $2 \epsilon$ of the "noise free" observations $A x_{0}$ thanks to the triangle inequality

$$
\left\|A x^{\sharp}-A x_{0}\right\|_{\ell_{2}} \leq\left\|A x^{\sharp}-y\right\|_{\ell_{2}}+\left\|A x_{0}-y\right\|_{\ell_{2}} \leq 2 \epsilon .
$$

Geometrically, this says that $x^{\sharp}$ is known to be in a cylinder around the $n$-dimensional plane $A x_{0}$.

2. Cone constraint. Since $x_{0}$ is feasible, we must have $\left\|x^{\sharp}\right\|_{\ell_{1}} \leq\left\|x_{0}\right\|_{\ell_{1}}$. Decompose $x^{\sharp}$ as $x^{\sharp}=x_{0}+h$. As observed in [13]

$$
\left\|x_{0}\right\|_{\ell_{1}}-\left\|h_{T_{0}}\right\|_{\ell_{1}}+\left\|h_{T_{0}^{c}}\right\|_{\ell_{1}} \leq\left\|x_{0}+h\right\|_{\ell_{1}} \leq\left\|x_{0}\right\|_{\ell_{1}},
$$

where $T_{0}$ is the support of $x_{0}$, and $h_{T_{0}}(t)=h(t)$ for $t \in T_{0}$ and zero elsewhere (similarly for $h_{T_{0}^{c}}$ ). Hence, $h$ obeys the cone constraint

$$
\left\|h_{T_{0}^{c}}\right\|_{\ell_{1}} \leq\left\|h_{T_{0}}\right\|_{\ell_{1}}
$$




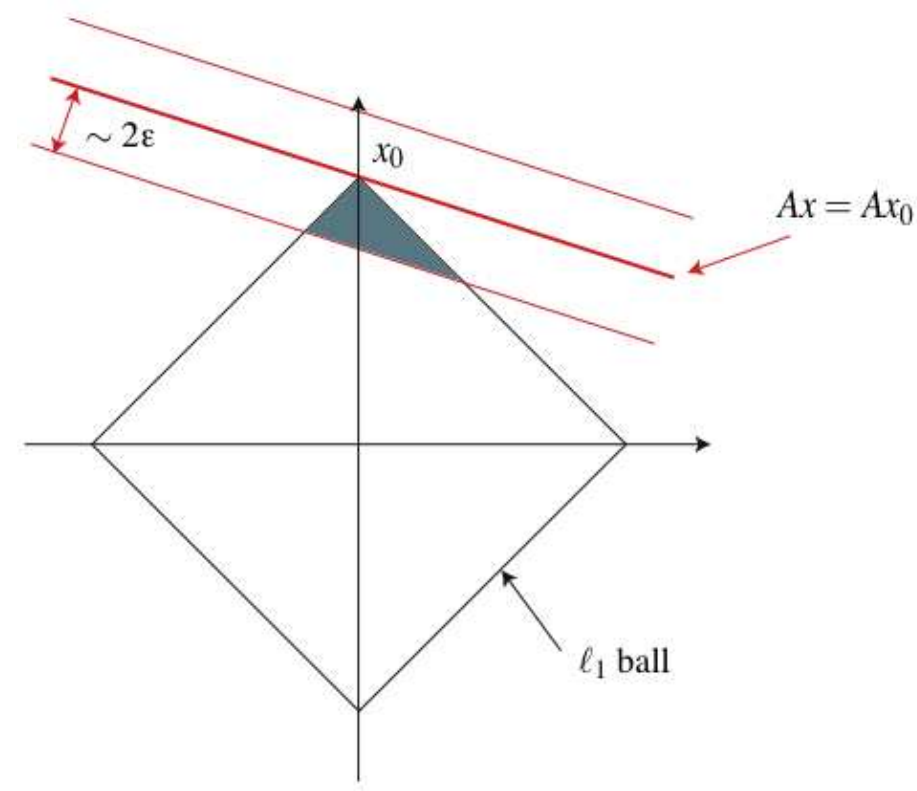

Figure 1: Geometry in $\mathbb{R}^{2}$. Here, the point $x_{0}$ is a vertex of the $\ell_{1}$ ball and the shaded area represents the set of points obeying both the tube and the cone constraints. By showing that every vector in the cone of descent at $x_{0}$ is approximately orthogonal to the nullspace of $A$, we will ensure that $x^{\sharp}$ is not too far from $x_{0}$.

which expresses the geometric idea that $h$ must lie in the cone of descent of the $\ell_{1}$-norm at $x_{0}$.

Figure 1illustrates both these geometrical constraints. Stability follows from the fact that the intersection between (9) $\left(\|A h\|_{\ell_{2}} \leq 2 \epsilon\right)$ and (10) is a set with small radius. The reason why this holds is because every vector $h$ in the $\ell_{1}$-cone (10) is approximately orthogonal to the nullspace of $A$. We shall prove that $\|A h\|_{\ell_{2}} \approx\|h\|_{\ell_{2}}$ and together with (9), this establishes the theorem.

We begin by dividing $T_{0}^{c}$ into subsets of size $M$ (we will choose $M$ later) and enumerate $T_{0}^{c}$ as $n_{1}, n_{2}, \ldots, n_{N-\left|T_{0}\right|}$ in decreasing order of magnitude of $h_{T_{0}^{c}}$. Set $T_{j}=\left\{n_{\ell},(j-1) M+1 \leq\right.$ $\ell \leq j M\}$. That is, $T_{1}$ contains the indices of the $M$ largest coefficients of $h_{T_{0}^{c}}, T_{2}$ contains the indices of the next $M$ largest coefficients, and so on.

With this decomposition, the $\ell_{2}$-norm of $h$ is concentrated on $T_{01}=T_{0} \cup T_{1}$. Indeed, the $k$ th largest value of $h_{T_{0}^{c}}$ obeys

$$
\left|h_{T_{0}^{c}}\right|_{(k)} \leq\left\|h_{T_{0}^{c}}\right\|_{\ell_{1}} / k
$$

and, therefore,

$$
\left\|h_{T_{01}^{c}}\right\|_{\ell_{2}}^{2} \leq\left\|h_{T_{0}^{c}}\right\|_{\ell_{1}}^{2} \sum_{k=M+1}^{m} 1 / k^{2} \leq\left\|h_{T_{0}^{c}}\right\|_{\ell_{1}}^{2} / M .
$$

Further, the $\ell_{1}$-cone constraint gives

$$
\left\|h_{T_{01}^{c}}\right\|_{\ell_{2}}^{2} \leq\left\|h_{T_{0}}\right\|_{\ell_{1}}^{2} / M \leq\left\|h_{T_{0}}\right\|_{\ell_{2}}^{2} \cdot\left|T_{0}\right| / M
$$


and thus

$$
\|h\|_{\ell_{2}}^{2}=\left\|h_{T_{01}}\right\|_{\ell_{2}}^{2}+\left\|h_{T_{01}^{c}}\right\|_{\ell_{2}}^{2} \leq\left(1+\left|T_{0}\right| / M\right) \cdot\left\|h_{T_{01}}\right\|_{\ell_{2}}^{2}
$$

Observe now that

$$
\begin{aligned}
\|A h\|_{\ell_{2}}=\left\|A_{T_{01}} h_{T_{01}}+\sum_{j \geq 2} A_{T_{j}} h_{T_{j}}\right\|_{\ell_{2}} & \geq\left\|A_{T_{01}} h_{T_{01}}\right\|_{\ell_{2}}-\left\|\sum_{j \geq 2} A_{T_{j}} h_{T_{j}}\right\|_{\ell_{2}} \\
& \geq\left\|A_{T_{01}} h_{T_{01}}\right\|_{\ell_{2}}-\sum_{j \geq 2}\left\|A_{T_{j}} h_{T_{j}}\right\|_{\ell_{2}} \\
& \geq \sqrt{1-\delta_{M+\left|T_{0}\right|}}\left\|h_{T_{01}}\right\|\left\|_{\ell_{2}}-\sqrt{1+\delta_{M}} \sum_{j \geq 2}\right\| h_{T_{j}} \|_{\ell_{2}} .
\end{aligned}
$$

Set $\rho=\left|T_{0}\right| / M$. As we shall see later,

$$
\sum_{j \geq 2}\left\|h_{T_{j}}\right\|_{\ell_{2}} \leq \sqrt{\rho} \cdot\left\|h_{T_{0}}\right\|_{\ell_{2}}
$$

which gives

$$
\|A h\|_{\ell_{2}} \geq C_{\left|T_{0}\right|, M} \cdot\left\|h_{T_{01}}\right\|_{\ell_{2}}, \quad C_{\left|T_{0}\right|, M}:=\sqrt{1-\delta_{M+\left|T_{0}\right|}}-\sqrt{\rho} \sqrt{1+\delta_{M}} .
$$

It then follows from (11) and $\|A h\|_{\ell_{2}} \leq 2 \epsilon$ that

$$
\|h\|_{\ell_{2}} \leq \sqrt{1+\rho} \cdot\left\|h_{T_{01}}\right\|_{\ell_{2}} \leq \frac{\sqrt{1+\rho}}{C_{\left|T_{0}\right|, M}} \cdot\|A h\|_{\ell_{2}} \leq \frac{2 \sqrt{1+\rho}}{C_{\left|T_{0}\right|, M}} \cdot \epsilon
$$

provided that the denominator is of course positive.

We may specialize (14) and take $M=3\left|T_{0}\right|$. The denominator is positive if $\delta_{3\left|T_{0}\right|}+3 \delta_{4\left|T_{0}\right|}<2$ (this is true if $\delta_{4\left|T_{0}\right|}<1 / 2$, say) which proves the theorem. Note that if $\delta_{4 S}$ is a little smaller, the constant in (5) is not large. For $\delta_{4 S} \leq 1 / 5, C_{S} \approx 8.82$, while for $\delta_{4 S} \leq 1 / 4, C_{S} \approx 10.47$ as claimed.

It remains to argue about (12). Observe that by construction, the magnitude of each coefficient in $T_{j+1}$ is less than the average of the magnitudes in $T_{j}$ :

$$
\left|h_{T_{j+1}}(t)\right| \leq\left\|h_{T_{j}}\right\|_{\ell_{1}} / M
$$

Then

$$
\left\|h_{T_{j+1}}\right\|_{\ell_{2}}^{2} \leq\left\|h_{T_{j}}\right\|_{\ell_{1}}^{2} / M
$$

and (12) follows from

$$
\sum_{j \geq 2}\left\|h_{T_{j}}\right\|_{\ell_{2}} \leq \sum_{j \geq 1}\left\|h_{T_{j}}\right\|_{\ell_{1}} / \sqrt{M} \leq\left\|h_{T_{0}}\right\|_{\ell_{1}} / \sqrt{M} \leq \sqrt{\left|T_{0}\right| / M} \cdot\left\|h_{T_{0}}\right\|_{\ell_{2}} .
$$

\subsection{Proof of Theorem 2; general case}

Suppose now that $x_{0}$ is arbitrary. We let $T_{0}$ be the indices of the largest $\left|T_{0}\right|$ coefficients of $x_{0}$ (the value $\left|T_{0}\right|$ will be decided later) and just as before, we divide up $T_{0}^{c}$ into sets $T_{1}, \ldots, T_{J}$ of equal size $\left|T_{j}\right|=M, j \geq 1$, by decreasing order of magnitude. The cone 
constraint (10) may not hold but a variation does. Indeed, $x=x_{0}+h$ is feasible and, therefore,

$$
\left\|x_{0, T_{0}}\right\|_{\ell_{1}}-\left\|h_{T_{0}}\right\|_{\ell_{1}}-\left\|x_{0, T_{0}^{c}}\right\|_{\ell_{1}}+\left\|h_{T_{0}^{c}}\right\|_{\ell_{1}} \leq\left\|x_{0, T_{0}}+h_{T_{0}}\right\|_{\ell_{1}}+\left\|x_{0, T_{0}^{c}}+h_{T_{0}^{c}}\right\|_{\ell_{1}} \leq\left\|x_{0}\right\|_{\ell_{1}},
$$

which gives

$$
\left\|h_{T_{0}^{c}}\right\|_{\ell_{1}} \leq\left\|h_{T_{0}}\right\|_{\ell_{1}}+2\left\|x_{0, T_{0}^{c}}\right\|_{\ell_{1}} .
$$

The rest of the argument now proceeds essentially as before. First, $h$ is in the some sense concentrated on $T_{01}=T_{0} \cup T_{1}$ since with the same notations

$$
\left\|h_{T_{01}^{c}}\right\|_{\ell_{2}} \leq \frac{\left\|h_{T_{0}}\right\|_{\ell_{1}}+2\left\|x_{0, T_{0}^{c}}\right\|_{\ell_{1}}}{\sqrt{M}} \leq \sqrt{\rho} \cdot\left(\left\|h_{T_{0}}\right\|_{\ell_{2}}+\frac{2\left\|x_{0, T_{0}^{c}}\right\|_{\ell_{1}}}{\sqrt{\left|T_{0}\right|}}\right),
$$

which in turn implies

$$
\|h\|_{\ell_{2}} \leq(1+\sqrt{\rho})\left\|h_{T_{01}}\right\|_{\ell_{2}}+2 \sqrt{\rho} \cdot \eta, \quad \eta:=\left\|x_{0, T_{0}^{c}}\right\|_{\ell_{1}} / \sqrt{\left|T_{0}\right|} .
$$

Better estimates via Pythagoras' formula are of course possible (see (11)) but we ignore such refinements in order to keep the argument as simple as possible. Second, the same reasoning as before gives

$$
\sum_{j \geq 2}\left\|h_{T_{j}}\right\|_{\ell_{2}} \leq \frac{\left\|h_{T_{01}^{c}}\right\|_{\ell_{1}}}{\sqrt{M}} \leq \sqrt{\rho} \cdot\left(\left\|h_{T_{0}}\right\|_{\ell_{2}}+2 \eta\right)
$$

and thus

$$
\|A h\|_{\ell_{2}} \geq C_{\left|T_{0}\right|, M} \cdot\left\|h_{T_{01}}\right\|_{\ell_{2}}-2 \sqrt{\rho} \sqrt{1+\delta_{M}} \cdot \eta,
$$

where $C_{\left|T_{0}\right|, M}$ is the same as in (13). Since $\|A h\| \leq 2 \epsilon$, we again conclude that

$$
\left\|h_{T_{01}}\right\|_{\ell_{2}} \leq \frac{2}{C_{\left|T_{0}\right|, M}} \cdot\left(\epsilon+\sqrt{\rho} \sqrt{1+\delta_{M}} \eta\right)
$$

(note that the constant in front of the $\epsilon$ factor is the same as in the truly sparse case) and the claim (6) follows from (16). Specializing the bound to $M=3\left|T_{0}\right|$ and assuming that $\delta_{S} \leq 1 / 5$ gives the numerical values reported in the statement of the theorem.

\section{Numerical Examples}

This section illustrates the effectiveness of the recovery by means of a few simple numerical experiments. Our simulations demonstrate that in practice, the constants in (5) and (6) seem to be quite low.

Our first series of experiments is summarized in Tables 1 1 and 2. In each experiment, a length 1024 signal was measured with the same $300 \times 1024$ Gaussian measurement ensemble. The measurements were then corrupted by additive white Gaussian noise: $y_{k}=\left\langle x_{0}, a_{k}\right\rangle+e_{k}$ with $e_{k} \sim \mathcal{N}\left(0, \sigma^{2}\right)$ for various noise levels $\sigma$. The squared norm of the error $\|e\|_{\ell_{2}}^{2}$ is a chi-square random variable with mean $\sigma^{2} n$ and standard deviation $\sigma^{2} \sqrt{2 n}$; owing to well known concentration inequalities, the probability that $\|e\|_{\ell_{2}}^{2}$ exceeds its mean plus two or three standard deviations is small. We then solve $\left(P_{2}\right)$ with

$$
\epsilon^{2}=\sigma^{2}(n+\lambda \sqrt{2 n})
$$


and select $\lambda=2$ although other choices are of course possible.

Table 1 charts the results for sparse signals with 50 nonzero components. Ten signals were generated by choosing 50 indices uniformly at random, and then selecting either -1 or 1 at each location with equal probability. An example of such a signal is shown in Figure 2(a). Previous experiments [3] have demonstrated that we were empirically able to recover such signals perfectly from 300 noiseless Gaussian measurements, which is indeed the case for each of the 10 signals considered here. The average value of the recovery error (taken over the 10 signals) is recorded in the bottom row of Table 1. In this situation, the constant in (5) appears to be less than 2 .

Table 2 charts the results for 10 compressible signals whose components are all non-zero, but decay as in (77). The signals were generated by taking a fixed sequence

$$
x_{\mathrm{sort}}(t)=(5.819) \cdot t^{-10 / 9}
$$

randomly permuting it, and multiplying by a random sign sequence (the constant in (18) was chosen so that the norm of the compressible signals is the same $-\sqrt{50}-$ as the sparse signals in the previous set of experiments). An example of such a signal is shown in Figure 2(c). Again, 10 such signals were generated, and the average recovery error recorded in the bottom row of Table 2 .

For small values of $\sigma$, the recovery error is dominated by the approximation error - the second term on the right hand side of (6). As a reference, the 50 term nonlinear approximation errors of these compressible signals is around 0.47; at low signal-to-noise ratios our recovery error is about 1.5 times this quantity. As the noise power gets large, the recovery error becomes less than $\epsilon$, just as in the sparse case.

Finally, we apply our recovery procedure to realistic imagery. Photograph-like images, such as the $256 \times 256$ pixel Boats image shown in Figure B(a), have wavelet coefficient sequences that are compressible (see [7]). The image is a 65536 dimensional vector, making the standard Gaussian ensemble too unwieldy ${ }^{3}$. Instead, we make 25000 measurements of the image using a scrambled real Fourier ensemble; that is, the test functions $a_{k}(t)$ are real-valued sines and cosines (with randomly selected frequencies) which are temporally scrambled by randomly permuting the $m$ time points. In other words, this ensemble is obtained from the (real-valued) Fourier ensemble by a random permutation of the columns. For our purposes here, the test functions behave like a Gaussian ensemble in the sense that from $n$ measurements, one can recover signals with about $n / 5$ nonzero components exactly from noiseless data. There is a computational advantage as well, since we can apply $A$ and its adjoint $A^{T}$ to an arbitrary vector by means of an $m$ point FFT. To recover the wavelet coefficients of the object, we simply solve

$$
\left(P_{2}^{\prime}\right) \quad \min \|\alpha\|_{\ell_{1}} \text { subject to }\left\|A W^{*} \alpha-y\right\|_{\ell_{2}} \leq \epsilon,
$$

where $A$ is the scrambled Fourier ensemble, and $W$ is the discrete Daubechies- 8 orthogonal wavelet transform.

We will attempt to recover the image from measurements perturbed in two different manners. First, as in the 1D experiments, the measurements were corrupted by additive white Gaussian noise with $\sigma=5 \cdot 10^{-4}$ so that $\sigma \cdot \sqrt{n}=.0791$. As shown in Figure 4 , the noise level

\footnotetext{
${ }^{3}$ Storing a double precision $25000 \times 65536$ matrix would use around 13.1 gigabytes of memory, about the capacity of three standard DVDs.
} 
is significant; the signal-to-noise ratio is $\left\|A x_{0}\right\|_{\ell_{2}} /\|e\|_{\ell_{2}}=4.5$. With $\epsilon=.0798$ as in (17), the recovery error is $\left\|\alpha^{\sharp}-\alpha_{0}\right\|_{\ell_{2}}=0.1303$ (the original image has unit norm). For comparison, the 5000 term nonlinear approximation error for the image is $\left\|\alpha_{0,5000}-\alpha_{0}\right\|_{\ell_{2}}=0.050$. Hence the recovery error is very close to the sum of the approximation error and the size of the perturbation.

Another type of perturbation of practical interest is round-off or quantization error. In general, the measurements cannot be taken with arbitrary precision, either because of limitations inherent to the measuring device, or that we wish to communicate them using some small number of bits. Unlike additive white Gaussian noise, round-off error is deterministic and signal dependent - a situation our methodology deals with easily.

The round-off error experiment was conducted as follows. Using the same scrambled Fourier ensemble, we take 25000 measurements of Boats, and round (quantize) them to one digit (we restrict the values of the measurements to be one of ten preset values, equally spaced). The measurement error is shown in Figure 4(c), and the signal-to-noise ratio is $\left\|A x_{0}\right\|_{\ell_{2}} /\|e\|_{\ell_{2}}=4.3$. To choose $\epsilon$, we use a rough model for the size of the perturbation. To a first approximation, the round-off error for each measurement behaves like a uniformly distributed random variable on $(-q / 2, q / 2)$, where $q$ is the distance between quantization levels. Under this assumption, the size of the perturbation $\|e\|_{\ell_{2}}^{2}$ would behave like a sum of squares of uniform random variables

$$
Y=\sum_{k=1}^{n} X_{k}^{2}, \quad X_{k} \sim \text { Uniform }\left(-\frac{q}{2}, \frac{q}{2}\right) .
$$

Here, mean $(Y)=n q^{2} / 12$ and $\operatorname{std}(Y)=\sqrt{n} q^{2} /[6 \sqrt{5}]$. Again, $Y$ is no larger than mean $(Y)+$ $\lambda \operatorname{std}(Y)$ with high probability, and we select

$$
\epsilon^{2}=n q^{2} / 12+\lambda \sqrt{n} q^{2} /[6 \sqrt{5}],
$$

where as before, $\lambda=2$. The results are summarized in the second column of Table 3. As in the previous case, the recovery error is very close to the sum of the approximation and measurement errors. Also note that despite the crude nature of the perturbation model, an accurate value of $\epsilon$ is chosen.

Although the errors in the recovery experiments summarized in the third row of Table 3 are as we hoped, the recovered images tend to contain visually displeasing high frequency oscillatory artifacts. To address this problem, we can solve a slightly different optimization problem to recover the image from the same corrupted measurements. In place of $\left(P_{2}^{\prime}\right)$, we solve

$$
(T V) \quad \min \|x\|_{T V} \quad \text { subject to } \quad\|A x-y\|_{\ell_{2}} \leq \epsilon
$$

where

$$
\|x\|_{T V}=\sum_{i, j} \sqrt{\left(x_{i+1, j}-x_{i, j}\right)^{2}+\left(x_{i, j+1}-x_{i, j}\right)^{2}}=\sum_{i, j}\left|(\nabla x)_{i, j}\right|
$$

is the total variation [16] of the image $x$ : the sum of the magnitudes of the (discretized) gradient. By substituting $(T V)$ for $\left(P_{2}^{\prime}\right)$, we are essentially changing our model for photographlike images. Instead of looking for an image with a sparse wavelet transform that explains the observations, program $(T V)$ searches for an image with a sparse gradient (i.e. without spurious high frequency oscillations). In fact, it is shown in [3] that just as signals which are exactly sparse can be recovered perfectly from a small number of measurements by solving 
Table 1: Recovery results for sparse 1D signals. Gaussian white noise of variance $\sigma^{2}$ was added to each of the $n=300$ measurements, and $\left(P_{2}\right)$ was solved with $\epsilon$ chosen such that $\|e\|_{2} \leq \epsilon$ with high probability (see (17)).

\begin{tabular}{|c||c|c|c|c|c|c|}
\hline$\sigma$ & 0.01 & 0.02 & 0.05 & 0.1 & 0.2 & 0.5 \\
\hline$\epsilon$ & 0.19 & 0.37 & 0.93 & 1.87 & 3.74 & 9.34 \\
\hline \hline$\left\|x^{\sharp}-x_{0}\right\|_{2}$ & 0.25 & 0.49 & 1.33 & 2.55 & 4.67 & 6.61 \\
\hline
\end{tabular}

Table 2: Recovery results for compressible 1D signals. Gaussian white noise of variance $\sigma^{2}$ was added to each measurement, and $\left(P_{2}\right)$ was solved with $\epsilon$ as in (17).

\begin{tabular}{|c||c|c|c|c|c|c|}
\hline$\sigma$ & 0.01 & 0.02 & 0.05 & 0.1 & 0.2 & 0.5 \\
\hline$\epsilon$ & 0.19 & 0.37 & 0.93 & 1.87 & 3.74 & 9.34 \\
\hline \hline$\left\|x^{\sharp}-x_{0}\right\|_{2}$ & 0.69 & 0.76 & 1.03 & 1.36 & 2.03 & 3.20 \\
\hline
\end{tabular}

$\left(P_{2}\right)$ with $\epsilon=0$, signals with gradients which are exactly sparse can be recovered by solving $(T V)$ (again with $\epsilon=0$ ).

Figure 3(b) and (c) and the fourth row of Table 3 show the $(T V)$ recovery results. The reconstructions have smaller error and do not contain visually displeasing artifacts.

\section{Discussion}

The convex programs $\left(P_{2}\right)$ and $(T V)$ are simple instances of a class of problems known as second-order cone programs (SOCP's). As an example, one can recast $(T V)$ as

$$
\min \sum_{i, j} u_{i, j} \quad \text { subject to } \quad-u_{i, j} \leq\left\|G_{i, j} x\right\|_{\ell_{2}} \leq u_{i, j},\|A x-b\|_{\ell_{2}} \leq \epsilon,
$$

where $G_{i, j} x=\left(x_{i+1, j}-x_{i, j}, x_{i, j+1}-x_{i, j}\right)$ [15]. SOCP's can nowadays be solved efficiently by interior-point methods [1] and, hence, our approach is computationally tractable.

From a certain viewpoint, recovering via $\left(P_{2}\right)$ is using a priori information about the nature of the underlying image, i.e. that it is sparse in some known orthobasis, to overcome the shortage of data. In practice, we could of course use far more sophisticated models to perform the recovery. Obvious extensions include looking for signals that are sparse

Table 3: Image recovery results. Measurements of the Boats image were corrupted in two different ways: by adding white noise (left column) with $\sigma=5 \cdot 10^{-4}$ and by rounding off to one digit (right column). In each case, the image was recovered in two different ways: by solving $\left(P_{2}^{\prime}\right)$ (third row) and solving $(T V)$ (fourth row). The $(T V)$ images are shown in Figure 3

\begin{tabular}{|c||c|c|}
\hline & White noise & Round-off \\
\hline \hline$\|e\|_{\ell_{2}}$ & 0.0789 & 0.0824 \\
\hline$\epsilon$ & 0.0798 & 0.0827 \\
\hline \hline$\left\|\alpha^{\sharp}-\alpha_{0}\right\|_{\ell_{2}}$ & 0.1303 & 0.1323 \\
\hline$\left\|\alpha_{T V}^{\sharp}-\alpha_{0}\right\|_{\ell_{2}}$ & 0.0837 & 0.0843 \\
\hline
\end{tabular}




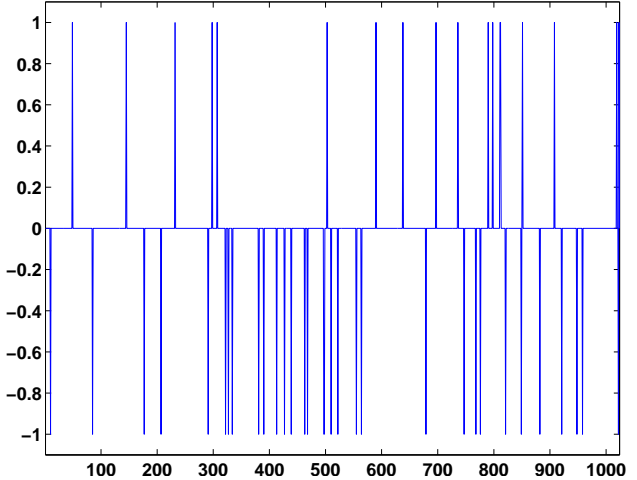

(a)

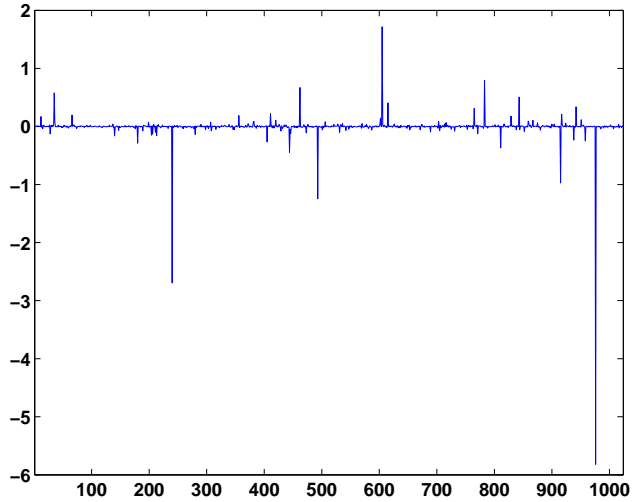

(c)

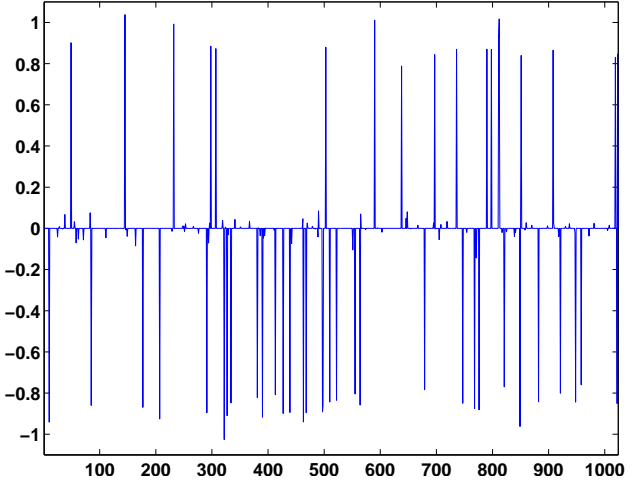

(b)

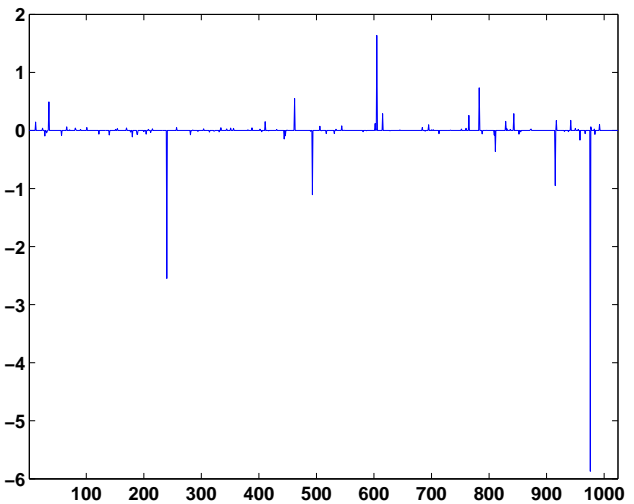

(d)

Figure 2: (a) Example of a sparse signal used in the $1 D$ experiments. There are 50 non-zero coefficients taking values \pm 1 . (b) Sparse signal recovered from noisy measurements with $\sigma=0.05$. (c) Example of a compressible signal used in the $1 D$ experiments. (d) Compressible signal recovered from noisy measurements with $\sigma=0.05$.

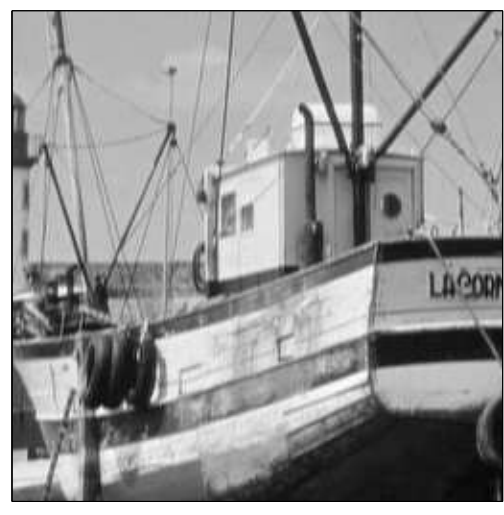

(a)

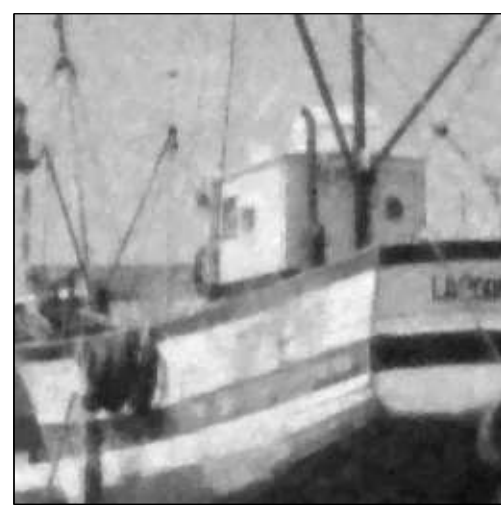

(b)

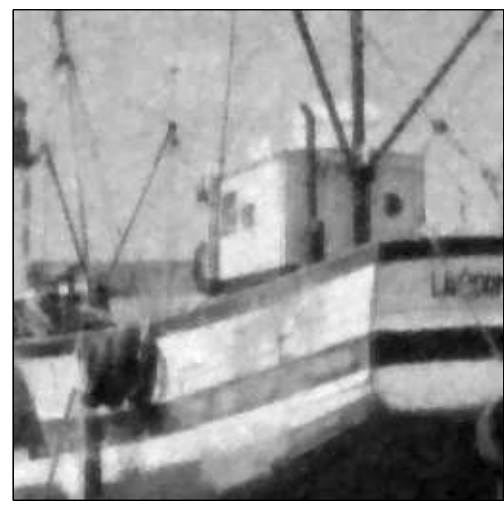

(c)

Figure 3: (a) Original $256 \times 256$ Boats image. (b) Recovery via (TV) from $n=25000$ measurements corrupted with Gaussian noise. (c) Recovery via (TV) from $n=25000$ measurements corrupted by round-off error. In both cases, the reconstruction error is less than the sum of the nonlinear approximation and measurement errors. 


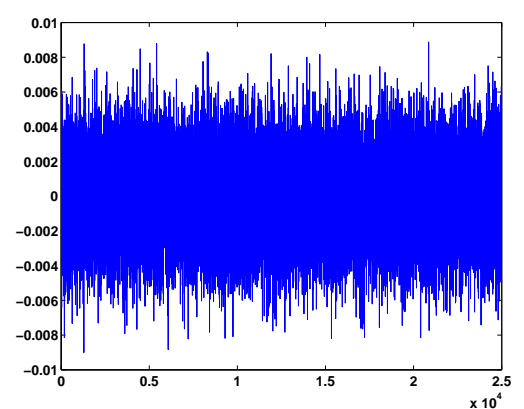

(a)

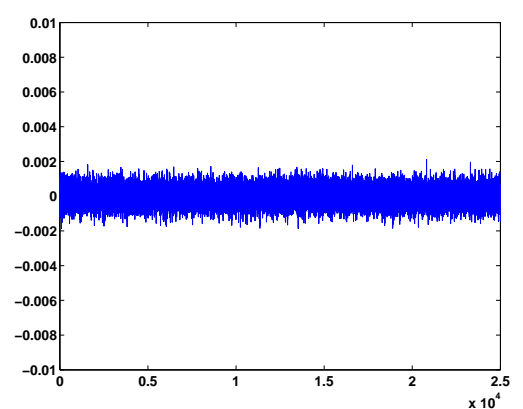

(b)

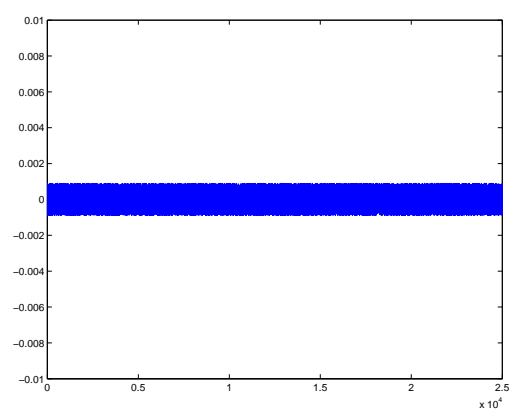

(c)

Figure 4: (a) Noiseless measurements $A x_{0}$ of the Boats image. (b) Gaussian measurement error with $\sigma=5 \cdot 10^{-4}$ in the recovery experiment summarized in the left column of Table 3 The signalto-noise ratio is $\left\|A x_{0}\right\|_{\ell_{2}} /\|e\|_{\ell_{2}}=4.5$. (c) Round-off error in the recovery experiment summarized in the right column of Table 3. The signal-to-noise ratio is $\left\|A x_{0}\right\|_{\ell_{2}} /\|e\|_{\ell_{2}}=4.3$.

in overcomplete wavelet or curvelet bases, or for images that have certain geometrical structure. The numerical experiments in Section 3 show how changing the model can result in a higher quality recovery from the same set of measurements.

\section{References}

[1] S. Boyd and L. Vandenberghe. Convex Optimization. Cambridge University Press, 2004 .

[2] E. J. Candès and J. Romberg. Quantitative robust uncertainty principles and optimally sparse decompositions. submitted to Foundations of Comput. Math., November 2004. Available on the ArXiv preprint server:math.CA/0411273.

[3] E. J. Candès, J. Romberg, and T. Tao. Robust uncertainty principles: Exact signal reconstruction from highly incomplete frequency information. submitted to IEEE Trans. Inform. Theory, June 2004. Available on theArXiV preprint server: math. GM/0409186.

[4] E. J. Candès and T. Tao. Decoding by linear programming. submitted to IEEE Trans. Inform. Theory, December 2004.

[5] E. J. Candès and T. Tao. Near-optimal signal recovery from random projections and universal encoding strategies. submitted to IEEE Trans. Inform. Theory, November 2004. Available on the ArXiV preprint server: math.CA/0410542.

[6] S. S. Chen, D. L. Donoho, and M. A. Saunders. Atomic decomposition by basis pursuit. SIAM J. Sci. Comput., 20:33-61, 1999.

[7] R. A. DeVore, B. Jawerth, and B. J. Lucier. Image compression through wavelet transform coding. IEEE Trans. Inform. Theory, 38(2):719-746, March 1992.

[8] D. L. Donoho. Compressed sensing. submitted to IEEE Trans. Inform. Theory, September 2004 .

[9] D. L. Donoho. For most large undetermined systems of linear equations the minimal $\ell_{1}$-norm near-solution is also the sparsest near-solution. Manuscript, September 2004. 
[10] D. L. Donoho. For most large undetermined systems of linear equations the minimal $\ell_{1}$-norm solution is also the sparsest solution. Manuscript, September 2004.

[11] D. L. Donoho and M. Elad. Optimally sparse representation in general (nonorthogonal) dictionaries via $\ell_{1}$ minimization. Proc. Natl. Acad. Sci. USA, 100:2197$2202,2003$.

[12] D. L. Donoho, M. Elad, and V. Temlyakov. Stable recovery of sparse overcomplete representations in the presence of noise. submitted to IEEE Trans. Inform. Theory, February 2004.

[13] D. L. Donoho and X. Huo. Uncertainty principles and ideal atomic decomposition. IEEE Trans. Inform. Theory, 47:2845-2862, 2001.

[14] A. C. Gilbert, S. Muthukrishnan, and M. Strauss. Beating the $b^{2}$ bottleneck in estimating $b$-term Fourier representations. Manuscript, May 2004.

[15] D. Goldfarb and W. Yin. Second-order cone programming based methods for total variation image restoration. Technical report, Columbia University, 2004. Submitted to SIAM J. on Scientific Computing.

[16] L. I. Rudin, S. Osher, and E. Fatemi. Nonlinear total variation noise removal algorithm. Physica D, 60:259-68, 1992.

[17] S. J. Szarek. Condition numbers of random matrices. J. Complexity, 7:131-149, 1991.

[18] J. Tropp. Just relax: Convex programming methods for identifying sparse signals in noise. submitted to IEEE Trans. Inform. Theory, 2005.

[19] J. Zou, A. C. Gilbert, M. J. Strauss, and I. Daubechies. Theoretical and experimental analysis of a randomized algorithm for sparse Fourier transform analysis. Preprint, November 2004. Available on the ArXiV preprint server: math. NA/0411102. 